\section{TALENs and ZFNs are associated with different mutation signatures}

To the Editor: Zinc-finger nucleases (ZFNs) and transcription activator-like effector nucleases (TALENs) are of great interest for genome engineering in higher eukaryotic cells and organisms ${ }^{1-5}$. These enzymes contain the same FokI nuclease domain and induce site-specific DNA cleavage; the repair of this broken DNA via error-prone nonhomologous end joining gives rise to small insertions and deletions at the cleavage site, often disrupting genetic information. We have observed that, despite their similarities, ZFNs and TALENs are associated with different mutation patterns. We first compared ZFN and TALEN mutation signatures reported in the literature. We calculated the frequencies of insertions, deletions and complex patterns that include both insertions and deletions at target sites in mammalian cells, mammalian and nonmammalian organisms, and plants. Our analysis included a total of 1,456 mutant sequences at 122 target sites reported in 43 independent studies (Supplementary Table 1).

In all the systems that we examined, ZFN-induced mutations were much more evenly distributed between deletions and insertions than were TALEN-induced mutations (Fig. 1a). In particular, insertions were rarely obtained with TALENs. For example, ZFNs induced insertions in mammalian cells at a frequency of $43 \%$, comparable to that of deletions (52\%). In sharp contrast, TALENs induced insertions at a frequency of $1.6 \%$, much lower than that of deletions ( $89 \%$ ). In all other systems combined, the ZFN-induced insertion frequency was much higher than the TALEN-induced insertion frequency ( $24 \%$ vs. $4.1 \%$ ) (Student's $t$-test, $P<0.05)$. ZFNs were associated with lower deletion frequencies $(59 \%)$ than TALENs $(81 \%)(P<0.05)$. Complex patterns were obtained at comparable frequencies $(17 \%$ with ZFNs and $15 \%$ with TALENs).

As an independent test, we transfected human embryonic kidney 293 cells with plasmids encoding TALENs and ZFNs with overlapping target sites and then compared mutation patterns. In line with our analysis of the literature, TALENs, unlike ZFNs, rarely produced insertions (Fig. 1b and Supplementary Fig. 1). Five TALENs induced insertions at an overall frequency of $2.6 \%$, whereas ZFNs induced insertions much more frequently (39\%) $(P<0.05)$. We conclude that the differential mutation signatures induced by TALENs and ZFNs do not arise from differences in target loci.

We speculate that the larger size of spacers in the TALEN sites (12-21 base pairs (bp) $)^{5}$ compared to the ZFN sites (mostly 5-6 bp) might cause the difference in mutation patterns. The larger spacers in TALEN sites may give rise to heterogeneous overhangs when cleaved by TALENs. In contrast, ZFNs that target sites with 5- or 6-bp spacers produce defined 4- or 5-bp overhangs, which are efficiently filled in before ligation to yield insertions. Defined overhangs may facilitate targeted insertions of plasmid DNA at genomic sites ${ }^{6}$.
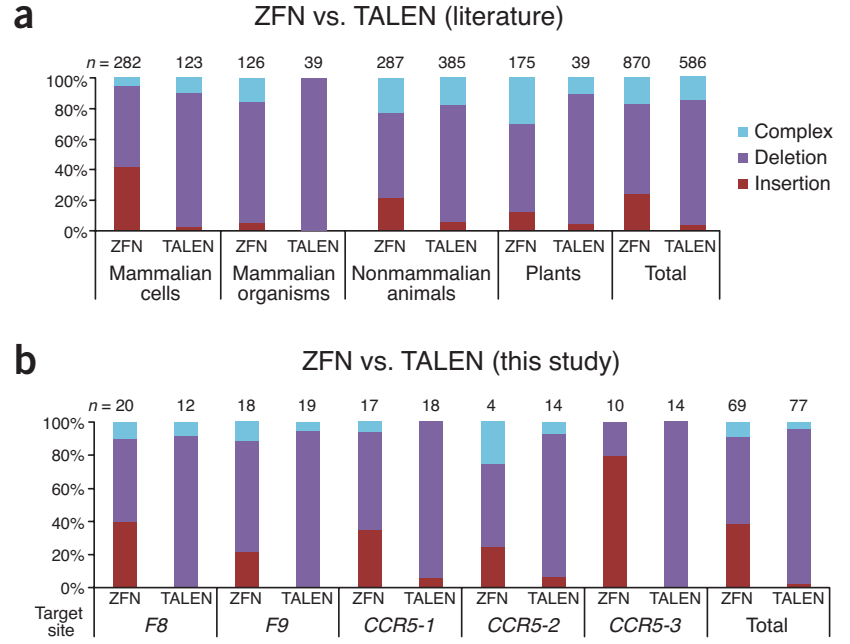

Figure 1 | Comparison of ZFN and TALEN mutation signatures. Nuclease-induced mutant sequences were classified as three different types: insertions, deletions or complex (that is, having both insertions and deletions) patterns. (a) ZFN and TALEN mutation patterns reported in the literature. (b) Comparison of ZFNs and TALENs that target overlapping sites. The number of mutant events is shown above each bar.

Our study shows that ZFNs and TALENs can produce different types of mutations, although whether this generalizes to all systems remains to be determined. Insertions and deletions could have different phenotypic consequences. Furthermore, organisms with insertions could be treated differently from those with deletions by regulatory authorities with regard to being labeled as genetically modified.

Note: Supplementary information is available at http://www.nature.com/ doifinder/10.1038/nmeth.2364.

\section{ACKNOWLEDGMENTS}

J.-S.K. is supported by the National Research Foundation of Korea (20120001225). We thank D. Carroll for helpful discussion.

\section{COMPETING FINANCIAL INTERESTS}

The authors declare no competing financial interests.

\section{Yongsub Kim ${ }^{1-3}$, Jiyeon Kweon ${ }^{1-3}$ \& Jin-Soo Kim ${ }^{1,2}$}

${ }^{1}$ National Creative Research Initiatives Center for Genome Engineering, Seoul National University, Seoul, South Korea. ${ }^{2}$ Department of Chemistry, Seoul National University, Seoul, South Korea. ${ }^{3}$ These authors contributed equally to this work.

e-mail: jskim01@snu.ac.kr

\section{PUBLISHED ONLINE 10 FEBRUARY 2013; DOI:10.1038/NMETH.2364}

1. Bibikova, M., Beumer, K., Trautman, J.K. \& Carroll, D. Science 300, 764 (2003).

2. Urnov, F.D. et al. Nature 435, 646-651 (2005).

3. Kim, H.J., Lee, H.J., Kim, H., Cho, S.W. \& Kim, J.S. Genome Res. 19, 1279-1288 (2009).

4. Kim, S., Lee, M.J., Kim, H., Kang, M. \& Kim, J.S. Nat. Methods 8, 7 (2011).

5. Miller, J.C. et al. Nat. Biotechnol. 29, 143-148 (2011).

6. Maresca, M., Lin, V.G., Guo, N. \& Yang, Y. Genome Res. published online, doi:10.1101/gr.145441.112 (14 November 2012). 